\title{
Postoperative Bleeding After Administration of a Single Dose of Rivaroxaban to a Patient Receiving Antiretroviral Therapy
}

\author{
Carmela E. Corallo $^{1}$ Louise Grannell ${ }^{1} \cdot$ Huyen Tran $^{2}$
}

Published online: 1 August 2015

(c) The Author(s) 2015. This article is published with open access at Springerlink.com

\begin{abstract}
A 62-year-old man was admitted to hospital for elective revision of a left total hip arthroplasty. His history was significant for human immunodeficiency virus (HIV) infection for which he was taking the following antiretroviral agents (ARVs): etravirine, ritonavir, darunavir, raltegravir and tenofovir/emtricitabine. Rivaroxaban $10 \mathrm{mg}$ daily was commenced on the second postoperative day for venous thromboembolism (VTE) prophylaxis. Approximately $24 \mathrm{~h}$ later, the patient developed hypotension and anaemia, accompanied by thigh swelling due to bleeding at the surgical site. Fluid resuscitation was commenced with red cell transfusion. The prothrombin time (PT) was prolonged at $24.3(10.6-15.3) \mathrm{s}$, and a rivaroxaban level taken $24 \mathrm{~h}$ after administration was $75 \mathrm{ng} / \mathrm{mL}$. Rivaroxaban was ceased, the PT normalised within $24 \mathrm{~h}$ of stopping the drug, and the patient made an uneventful recovery. None of the other coadministered drugs are known to interact with rivaroxaban, or are likely to, based on their metabolic pathways. Rivaroxaban, a substrate for cytochrome P450 (CYP) 3A4 and P-glycoprotein (P-gp), is contraindicated in patients concomitantly treated with strong inhibitors of both these systems, e.g. protease inhibitors (PIs) such as ritonavir (based on in vitro data and a pharmacokinetic study in healthy volunteers). No published data are available on the PI darunavir, a moderate inhibitor; however, concomitant use with rivaroxaban should also be avoided. A prolonged PT and a rivaroxaban trough level greater than
\end{abstract}

Carmela E. Corallo

c.corallo@alfred.org.au

1 Department of Pharmacy, Alfred Health, Alfred Hospital, 55 Commercial Road, Melbourne, VIC 3004, Australia

2 Haemostasis/Thrombosis Unit, Haemophilia Centre, Alfred Health, Melbourne, VIC, Australia eight times that predicted from pharmacokinetic modelling suggests that bleeding was due to increased exposure to rivaroxaban, probably due to an interaction with ritonavir and darunavir. This is supported by a Drug Interaction Probability Scale (DIPS) score of 8. An interaction between a single dose of rivaroxaban and ARVs may be clinically significant; therefore, the patient's medication history should be extensively evaluated to identify any potential interactions.

\section{Key Points}

Drug interactions with rivaroxaban are a potential cause for serious adverse effects.

An interaction between a single dose of rivaroxaban and protease inhibitors may result in bleeding.

Extensive evaluation of the interaction profile is essential prior to adding rivaroxaban to antiretroviral therapy.

\section{Introduction}

A recent study by McDonald et al. showed that a large proportion of spontaneous reports of adverse events with rivaroxaban were associated with concomitant medicines, which may have increased the risk [1]. The authors concluded that there is a need for ongoing postmarketing surveillance of rivaroxaban, together with an increased awareness of the potential for drug interactions as a cause 
for serious adverse events. However, there was no mention of reports of interactions with antiretroviral agents (ARVs). We would like to describe a case report that illustrates the rapid effect of an interaction between the target-specific oral anticoagulant (TSOAC) rivaroxaban and ARVs.

\section{Case Report}

A 62-year-old male orthopedic surgical patient receiving long-term ARVs was commenced on enoxaparin $40 \mathrm{mg}$ subcutaneously daily for venous thromboembolism (VTE) prophylaxis 3 weeks preoperatively. Preadmission medications that were continued postoperatively included the ARVs, etravirine $200 \mathrm{mg}$ twice daily, ritonavir $100 \mathrm{mg}$ twice daily, darunavir $600 \mathrm{mg}$ twice daily, raltegravir $400 \mathrm{mg}$ twice daily and emtricitabine/tenofovir 300/200 mg daily; mirtazapine $45 \mathrm{mg}$ at night, aspirin $100 \mathrm{mg}$ in the morning, esomeprazole $20 \mathrm{mg}$ daily, valaciclovir $500 \mathrm{mg}$ daily, and oxycodone sustained-release $60 \mathrm{mg}$ twice daily. New medications that were commenced postoperatively included intravenous prophylactic antibiotics, meropenem $1000 \mathrm{mg}$ three times daily and linezolid $600 \mathrm{mg}$ twice daily for 1 week, and a single dose of antifungal therapy, oral fluconazole $400 \mathrm{mg}$. Pain was managed with pregabalin $75 \mathrm{mg}$ twice daily, ketamine intravenous infusion up to $16 \mathrm{mg} / \mathrm{h}$ and morphine patient-controlled analgesia. Postoperatively, enoxaparin was replaced with rivaroxaban $10 \mathrm{mg}$ daily. Twenty-four hours after concomitant administration of rivaroxaban with ARVs, the patient experienced profound hypotension and bleeding at the surgical site. At the time of bleeding, laboratory results showed a prolonged prothrombin time (PT) of 24.3 (10.6-15.3) s and a significantly elevated rivaroxaban trough level of $75 \mathrm{ng} / \mathrm{mL}$ (median plasma concentration $24 \mathrm{~h}$ after a $10 \mathrm{mg}$ dose has been reported to be $9 \mathrm{ng} / \mathrm{mL}$ ) [2]. Renal function was normal. Rivaroxaban was ceased and the patient was managed with fluid resuscitation, packed red blood cells, fresh frozen plasma and human prothrombin complex (Prothrombinex$\mathrm{VF}^{\circledR}$ ). No further bleeding occurred and $24 \mathrm{~h}$ later PT had normalized and the rivaroxaban level had decreased to $11 \mathrm{ng} / \mathrm{mL}$. Enoxaparin for VTE prophylaxis was commenced 1 week later.

\section{Discussion}

Rivaroxaban, a substrate for cytochrome P450 (CYP) 3A4 and $\mathrm{P}$-glycoprotein ( $\mathrm{P}$-gp), is contraindicated in patients concomitantly treated with strong inhibitors of both these systems, e.g. protease inhibitors (PIs) such as ritonavir [3]. The PI darunavir, a moderate CYP3A4 inhibitor, should also be avoided in patients receiving rivaroxaban $[4,5]$.
This recommendation is based on in vitro data and a pharmacokinetic study in healthy volunteers of rivaroxaban coadministered with drugs that share its metabolic pathway, e.g. ritonavir, midazolam [6]. Our patient was taking six ARVs, including the two PIs ritonavir and darunavir, and one non-nucleoside reverse-transcriptase inhibitor (NNRTI), etravirine (a moderate CYP3A4 inducer) [4]. No clinically significant interactions were expected to occur with the other ARVs, i.e. the integrase inhibitor, raltegravir and the nucleoside reverse-transcriptase inhibitors (NRTIs) emtricitabine/tenofovir [5].

After oral administration of a CYP3A4 inhibitor such as ritonavir, there is a rapid increase in the substrate plasma level, which is reversible, typically within 2-3 days of stopping the inhibitor [7]. Ritonavir is used as a pharmacokinetic booster and is included in most PI-based regimens at the lower dose of $100 \mathrm{mg}$ twice daily compared with the $600 \mathrm{mg}$ twice-daily regimen when used for its antiviral activity [4]. In this setting, ritonavir is a pharmacologic enhancer because it inhibits the metabolism of coadministered PIs, resulting in an increase in blood and tissue levels. Administration of a single dose of rivaroxaban $10 \mathrm{mg}$ to healthy volunteers taking steady-state ritonavir $600 \mathrm{mg}$ twice daily has been shown to increase the area under the curve (AUC) by $153 \%$ and the mean maximum concentration $\left(C_{\max }\right)$ by $55 \%$ [6]. No pharmacokinetic data are available on the impact of lower doses of ritonavir, i.e. $100 \mathrm{mg}$ twice daily coadministered with rivaroxaban. In addition, no published data are available on the clinical significance of the interaction potential of darunavir, but the recommendation to avoid concomitant administration with rivaroxaban is similar to ritonavir [5].

Since the registration of rivaroxaban in Australia, in 2008 to November 2014 no reports, in addition to the one presented here, have been submitted to the Australian regulatory authority for therapeutic goods - the Therapeutics Goods Administration (TGA)—on adverse effects resulting from an interaction between rivaroxaban and ARVs [8]. To our knowledge, there is only one published case describing clinically significant bleeding possibly associated with an interaction between rivaroxaban and ARVs, including ritonavir, darunavir and etravirine [9]; however, a causal relationship between the adverse event and the drug interaction is unclear.

The clinical significance of an interaction between rivaroxaban and etravirine, a moderate CYP3A4 inducer, is not known. Induction of CYP3A4 is not an instantaneous process and may take 2-3 weeks to reach steady state [10]. Our patient had been taking etravirine for a number of years, therefore maximal induction was already present. The concomitant administration of rivaroxaban and etravirine may have decreased rivaroxaban levels to some extent and may have attenuated its effect. 
Other drugs such as fluconazole may interact with rivaroxaban; however, it is a moderate inhibitor of CYP3A4 and, potentially, Bcrp (ABCG2). In the study by Mueck et al., only a modest increase in rivaroxaban blood level was observed after 4 days of pretreatment with fluconazole [6]. Our patient had only received one dose of fluconazole, therefore it is not likely to have had an impact on rivaroxaban levels.

None of the other coadministered drugs are known to, or are likely to, interact with rivaroxaban based on their metabolic pathway, i.e. ketamine, morphine.

For TSOACs, routine coagulation tests can be useful as screening tests to determine residual anticoagulant effect in certain clinical situations, such as the presence of bleeding [11]. The PT is the most sensitive assay for detecting rivaroxaban; however, it can be influenced by a number of factors, including hepatic impairment, sepsis, trauma with significant blood loss and laboratory PT reagent (thromboplastin) sensitivity [12].

The clinical relevance of TSOAC drug levels is unknown and should not be used to improve effectiveness [13]. However, in the presence of TSOAC-associated bleeding, specific drug levels should be performed, in conjunction with appropriate coagulation testing, to assess the contribution of excess drug to the bleeding event and to guide the need for intervention. In patients undergoing hip replacement surgery, the median rivaroxaban plasma concentration after a $10 \mathrm{mg}$ once-daily dose was $9 \mathrm{ng} / \mathrm{ml} 24 \mathrm{~h}$ after the dose, i.e. trough level [2].

In our patient, the presence of a trough rivaroxaban level more than eight times higher than that expected from published data, together with a prolonged PT, suggests that bleeding may have been associated with increased exposure to the anticoagulant [2]. The rapid onset of bleeding and subsequent normalization of PT upon discontinuation of rivaroxaban is consistent with the pharmacokinetics of the drug and an interaction with ritonavir, which has been described in vitro and in healthy volunteers, and also with darunavir, based on its metabolic pathway. This is supported by a Drug Interaction Probability Scale (DIPS) score of 8 , indicating a probable association [14].

\section{Conclusions}

Prescribers and pharmacists need to be alert to the potential for this clinically significant interaction to occur and to use the combination of TSOACs and ARV regimens containing PIs with caution. Due to the potentially serious consequences, for patients on ARVs it may be prudent to consider alternative agents for VTE prophylaxis, such as lowmolecular-weight heparin, until an extensive evaluation of the interaction profile has been performed. Specialized resources for checking interactions with ARVs are readily available [5].

Written informed consent was obtained from the patient prior to publication of this case report.

\section{Compliance with Ethical Standards}

Conflicts of interest Carmela Corallo, Louise Grannell and Huyen Tran declare they have no conflicts of interest.

Funding No financial support was received for the preparation of this manuscript.

Open Access This article is distributed under the terms of the Creative Commons Attribution-NonCommercial 4.0 International License (http://creativecommons.org/licenses/by-nc/4.0/), which permits any noncommercial use, distribution, and reproduction in any medium, provided you give appropriate credit to the original author(s) and the source, provide a link to the Creative Commons license, and indicate if changes were made.

\section{References}

1. McDonald CJ, Ellett LMK, Barratt JD, Caughey GE. A crosscountry comparison of rivaroxaban spontaneous adverse event reports and concomitant medicine use with the potential to increase the risk of harm. Drug Saf. 2014;37:1029-35.

2. Mueck W, Borris LC, Dahl OE, Haas S, Huisman MV, Kakkar $\mathrm{AK}$, et al. Population pharmacokinetics and pharmacodynamics of once- and twice-daily rivaroxaban for the prevention of venous thromboembolism in patients undergoing total hip replacement. Thromb Haemost. 2008;100:453-61.

3. Rivaroxaban $\left(\right.$ Xarelto $\left.^{\circledR}\right)$ product information. MIMS Online. http://www.mimsonline.com.au. Accessed 24 Jun 2015.

4. Egan G, Hughes CA, Ackman ML. Drug interactions between antiplatelet or novel oral anticoagulant medications and antiretroviral medications. Ann Pharmacother. 2014;48:734-40.

5. Drug interaction charts. Liverpool (UK): University of Liverpool. www.hiv-druginteractions.org. Accessed 24 Jun 2015.

6. Mueck W, Kubitza D, Becka M. Co-administration of rivaroxaban with drugs that share its elimination pathways: pharmacokinetic effects in healthy subjects. $\mathrm{Br} \mathrm{J}$ Clin Pharmacol. 2012;76:455-66.

7. Kwong LM, Tong LM. Drug interactions with rivaroxaban following total joint replacement surgery. Ann Pharmacother. 2012;46:1232-8.

8. Australian Therapeutic Goods Administration. Database of Adverse Events Notifications (DAEN). 2015. http://www.tga.gov. au/safety/daen.htm. Accessed 1 Mar 2015.

9. Lakatos B, Stoeckle M, Elzi L, Battegay M, Marzolini C. Gastrointestinal bleeding associated with rivaroxaban administration in a treated patient infected with human immunodeficiency virus. Swiss Med Wkly. 2014;144:w13906.

10. Jimenez-Nacher I, Alvarez E, Morello J, Rodriguez-Novoa, De Andres S, Soriano J. Approaches for understanding and predicting drug interactions in human immunodeficiency virus-infected patients. Expert Opin Drug Metab Toxicol. 2011;7:457-77.

11. Tran H, Joseph J, Young L, et al. New oral anticoagulants: a practical guide on prescription, laboratory testing and peril-procedural/bleeding management. Intern Med J. 2014;44:525-36.

12. Lindhoff-Last E, Ansell J, Spiro T, Samna MM. Laboratory testing of rivaroxaban in routine clinical practice; when, how, and which assays. Ann Med. 2013;45:423-9. 
13. Mani H, Kasper A, Lindhoff-Last E. Measuring the anticoagulant effects of target specific oral anticoagulants-reasons, methods and current limitations. J Thromb Thrombolysis. 2013;36:187-94.
14. Horn JR, Hansen PD, Chan LN. Proposal for a new tool to evaluate drug interaction cases. Ann Pharmacother. 2007;41:674-80 